\title{
DETECTION OF SOLAR FIVE-MINUTE OSCILLATIONS OF LOW DEGREE*
}

\author{
PHILIP H. SCHERRER and JOHN M. WILCOX \\ Institute for Plasma Research, Stanford University, Stanford, CA 94305, U.S.A. \\ J. CHRISTENSEN-DALSGAARD \\ National Center for Atmospheric Research, Boulder, CO 80307, U.S.A. \\ and \\ D. O. GOUGH \\ Institute of Astronomy, Madingley Road, Cambridge CB3 OHA, U.K.
}

\begin{abstract}
Solar five-minute oscillations of degree $l=3,4$, and 5 have been observed at Stanford, in the Doppler shift of the Fe 5124 line. The frequencies and amplitudes are in broad agreement with previous observations of modes with $l \leq 3$, though we note that there are some systematic discrepancies between the results of different observers.
\end{abstract}

\section{Introduction}

Whole-disk observations of solar five-minute oscillations have been reported by Claverie et al. (1979, 1981a, b), by Grec et al. (1980), and by Fossat et al. (1981), Grec et al. (1983), who measured Doppler shifts, and by Deubner (1981) and Woodard and Hudson (1983), who measured intensity variations. These observations are sensitive mainly to oscillation of degree $l=0,1,2$. In addition, current whole-disk Doppler observations can detect octupole modes, though with low sensitivity.

By reducing the spatial scale over which light from the solar disk is integrated, the maximum sensitivity can be moved towards larger $l$. Here we report Doppler observations that were made at Stanford, and which are differences between mean spectrum line shifts in light from a central portion of the disk and from an outer annulus. Severny et al. (1981) have already reported detecting five-minute oscillations with a similar, though different, filter geometry. On comparing the oscillation frequencies with those reported by Grec et al. (1983) we were able to identify modes with $l=3$. Once that was accomplished, it was then easy to identify modes with $l=4$ and $l=5$. Contributions to the signal from modes of higher degree are under investigation.

\section{The Observing Technique}

The observations made at Stanford use a technique suggested by Severny et al. (1976). A Babcock (1953) solar magnetograph, designed for measuring Zeeman splitting, has

\footnotetext{
* Proceedings of the 66th IAU Colloquium: Problems in Solar and Stellar Oscillations, held at the Crimean Astrophysical Observatory, U.S.S.R., 1-5 September, 1981.
} 
been converted into an instrument with equivalent sensitivity for measuring Doppler shifts. The measurement is made by observing a Fraunhofer line that has no Zeeman splitting $(g=0)$. In the Stanford instrument a central circular area of the solar disk, of radius $0.50 R_{\odot}$, is covered with a right-hand circular polarizer, and the annulus between radii $0.55 R_{\odot}$ and $0.80 R_{\odot}$ is covered with a left-hand polarizer. The transmitted light-beam enters the magnetograph which, as usual, measures the wavelength difference between right and left circularly polarized light. This is interpreted as the difference in the mean line-of-sight velocity between the central portion of the solar disk and the outer annulus. Details of the observing procedure are described by Dittmer (1978).

The sensitivity of these observations to modes of different degree can be characterized by spatial filters $S_{l}$. These are defined as the ratio between the signal amplitude and the root-mean-square amplitude over the entire solar surface of the quantity that is presumed to be measured. Spatial filters $S_{l}^{v}$ for Doppler velocity measurements have been tabulated by Christensen-Dalsgaard and Gough (1982) and plotted by ChristensenDalsgaard (1980), for both whole-disk and Stanford observations. These are mean filters, averaged over all spherical orders $m$ of the surface harmonics that factor from the oscillation eigenfunctions. Thus, granted that there is no strongly preferred orientation of the modes, they represent the expectation value of the actual filter for an observation of any particular modes of degree $l$. For whole-disk observations $S_{l}^{c}$ is
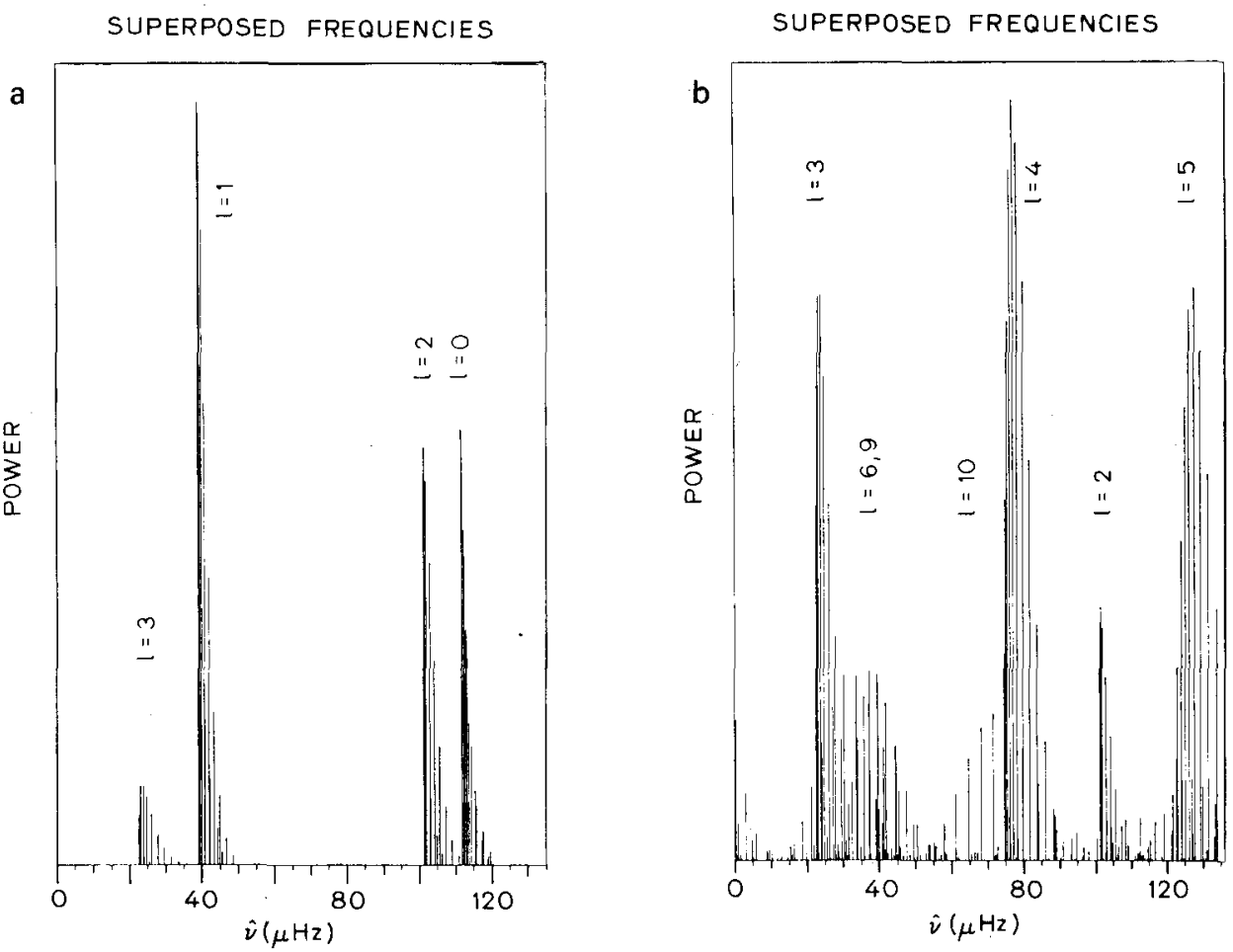

Fig. 1. Superposed frequency diagrams for model 1 with $\Delta v=136.2$ microHz: (a) for whole-disk filters, (b) for Stanford filters. 
largest when $l=1$, and is greater than $\frac{1}{2} S_{1}^{v}$ only for $l=0,1$, and 2 . On the other hand, for the Stanford geometry the largest value is for $l=4$, and $S_{l}^{v} \gtrsim \frac{1}{2} S_{4}^{v}$ for $l=2-6$ and also for $l=9$ and 10 . Thus, compared with whole-disk observations, the Stanford observations are sensitive to modes over a fairly wide range of $l$. Though this complicates the interpretation, such observations evidently increase the richness of the data.

\section{Anticipation of the Results, and Comparison with Whole-Disk Data}

The most pronounced difference between the results expected from the whole-disk and the Stanford observations can be illustrated with a superposed frequency diagram (Grec et al., 1980; Christensen-Dalsgaard, 1980). Each frequency $v$ is reduced to a frequency $\hat{v}$ lying in the range $\left(v_{0}, v_{0}+\Delta v\right)$ by adding or subtracting an integral multiple of $\Delta v$, which is characteristic of the separation in frequency between modes of adjacent order $n$ and of like degree $l$; the value of $v_{0}$ is arbitrary. The frequencies $\hat{v}(v)$ are plotted as a bar diagram, each bar having height $\left(S_{l}^{v}\right)^{2} \mathrm{~A}(v)$, where $A(v)$ is the expectation of the square of the amplitude of the mode; here we use the function that is defined by the first equation (D12) of Christensen-Dalsgaard and Gough (1982), which is a Gaussian approximation to the envelope of the power observed. The results are shown in Figures 1a and $1 \mathrm{~b}$, for whole-disk and Stanford filters, respectively. They represent idealized superposed power spectra, which one would obtain if the mode lifetimes and the observing interval were both infinite. In practice the observing interval is too short to enable the individual bars to be resolved, but it is easy to imagine how Figure 1 should be modified in that case. The striking resemblance between our Figure 1a and Figure 2 of Grec et al. (1980), which is a superposed power spectrum of $120^{\mathrm{h}}$ continuous whole-disk Doppler data obtained at South Pole, is the basis for the identification of the solar modes responsible for that data.

It is evident from Figure 1a that modes with $l=0,1$, and 2 dominate the whole-disk data, and that their frequencies are nearly uniformly spaced in $n$ with a spacing that depends only weakly on $l$. To each value of $l$ there corresponds a narrow range of frequencies $\hat{v}$; moreover the frequencies of the groups of modes with $l=0$ and $l=2$ (and with $l=1$ and $l=3$ ) are very close together. Thus in a power spectrum computed from observations limited to a single day, those closely spaced peaks merge; the spectrum has two peaks in each frequency interval $\Delta v$, as seen in the observations of Claverie $e t$ al. (1979). Adjacent peaks are expected to contain about the same power, and the peaks produced by modes with odd $l$ lie roughly midway between those with even $l$. Thus direct identification of the degrees of the modes producing a particular peak in such a spectrum is not possible.

In contrast Figure $1 \mathrm{~b}$ is dominated by nodes with $l=3,4$, and 5 , with substantial contributions from modes with $l=6$ and 9 , whose frequencies nearly coincide with those of the $l=3$ group, and modes with $l=2$ and 10 which are close to the $l=4$ group. Although the frequencies of modes with $l \gtrsim 3$ are still approximately uniformly spaced in $n$, the value of that separation increases more rapidly with $l$ (see Figure 7). Therefore the groups are more weakly confined. In spectra of a single day's observations, or 
averages of such spectra, one would expect three, rather than two, dominant peaks per frequency interval $\Delta v$, corresponding to modes with $l=3,4$, and 5 (cf. ChristensenDalsgaard and Gough, 1982), with perhaps some evidence for smaller peaks from other modes. Therefore the temporal resolution requirements are more stringent than for whole-disk observations. Once again the major peaks should be roughly equally spaced, with no obvious systematic variation in power from modes of different degree. Direct identification of $l$ from such data alone is therefore not possible.

\section{The Observations}

The observations were made in June and July, 1981, at the Stanford Solar Observatory. We obtained good quality data on 15 days; the intervals during which that data was acquired are listed in Table I. The average duration was $10.3 \mathrm{hr}$, and the shortest was $8.0 \mathrm{hr}$. The observations were made with a $0.1 \mathrm{~s}$ resolution but were averaged into $15 \mathrm{~s}$ intervals during the observations. The $15 \mathrm{~s}$ resolution was maintained throughout the subsequent analysis.

\section{TABLE I}

Times of observations (UT). The duration refers to the filtered data, and does not include the first and last $20 \mathrm{~min}$

\begin{tabular}{lllc}
\hline Date at start & Start & Stop & Duration (hr) \\
\hline 17 June 1981 & $13: 50$ & $01: 00$ & 10.5 \\
20 June & $16: 10$ & $02: 00$ & 9.3 \\
21 June & $18: 00$ & $02: 00$ & 7.3 \\
22 June & $14: 00$ & $22: 00$ & 7.3 \\
24 June & $16: 00$ & $01: 40$ & 9.0 \\
26 June & $16: 05$ & $01: 40$ & 8.9 \\
27 June & $15: 00$ & $01: 30$ & 9.8 \\
28 June & $16: 30$ & $01: 20$ & 8.2 \\
7 July & $14: 00$ & $02: 15$ & 11.6 \\
8 July & $14: 00$ & $01: 00$ & 10.3 \\
10 July & $14: 15$ & $02: 00$ & 11.1 \\
11 July & $14: 00$ & $02: 00$ & 11.3 \\
12 July & $13: 40$ & $02: 00$ & 11.7 \\
13 July & $14: 30$ & $22: 50$ & 8.3 \\
14 July & $15: 00$ & $01: 40$ & 10.0 \\
\hline
\end{tabular}

A typical day's observation is shown in Figure 2. Figure 2a is a plot of the line-of-sight Doppler velocity observed. It can be seen that a slow drift of about $1 \mathrm{~m} \mathrm{~s}^{-1}$ per hour is present. This was removed before analyzing the data by first applying a $10 \mathrm{~min}$ running mean four times, and then subtracting the smoothed data so obtained from the original. The result is shown in Figure $2 \mathrm{~b}$. With this kind of filtering the first and last 20 min of each data sequence must be discarded. Hence the average duration of filtered data that we analyzed was $9.6 \mathrm{hr}$. This is sufficient to resolve the major peaks in the power spectrum, whose mean separation we anticipated to be a little more than 


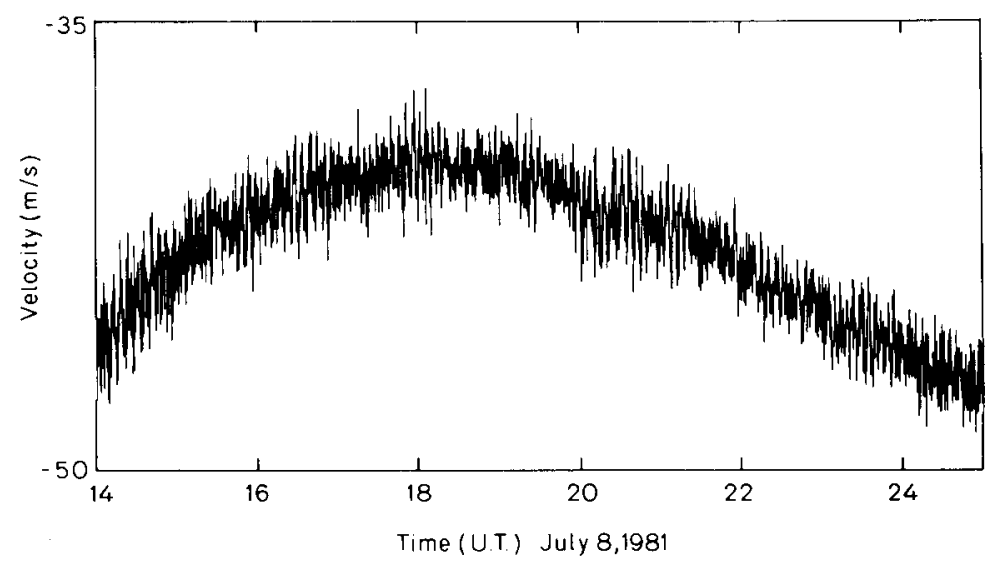

Fig. 2a. Solar velocity signal observed on a typical day, July 8,1981 . The drift through the day is caused by variations in sky transparency and by other unknown causes.

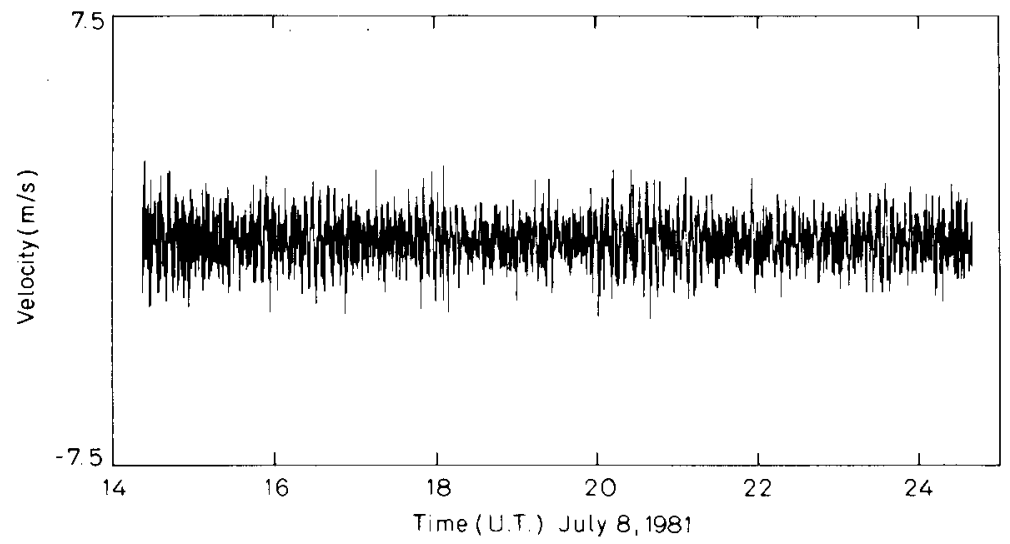

Fig. 2b. The same as Figure 2a after the filtering described in the text has been applied.

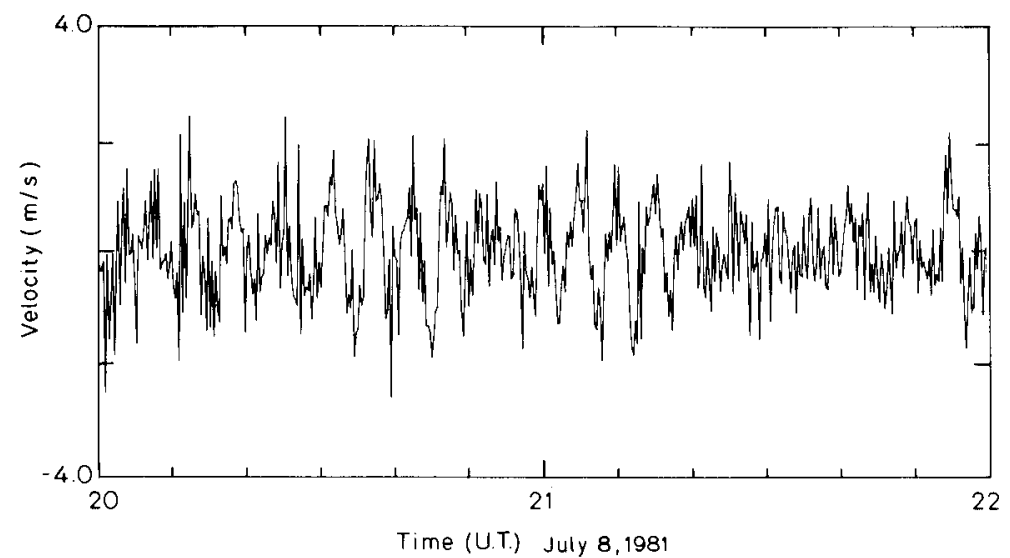

Fig. 2c. A typical two hour interval from Figure 2b. This is included to show that five minute oscillations are visible in the raw data. 
45 microHz. Figure $2 \mathrm{c}$ shows a $2 \mathrm{hr}$ interval from Figure $2 \mathrm{~b}$. Oscillation with periods near $5 \mathrm{~min}$ are clearly visible. The amplitude waxes and wanes as the various modes beat with each other.

\section{Analysis of the Data}

Harmonic amplitude spectra were computed for each day's observation by a direct least-squares method to find the Fourier coefficients in the frequency range $2.0 \mathrm{mHz}-4.5 \mathrm{mHz}$, in steps of $1.0 \mathrm{microHz}$. Figure 3 is the squared amplitude spectrum of the single day's data shown in Figure 2c. Figure 4 depicts the average of the 15 equally weighted spectra. Also included, for comparison, are the frequencies identified by

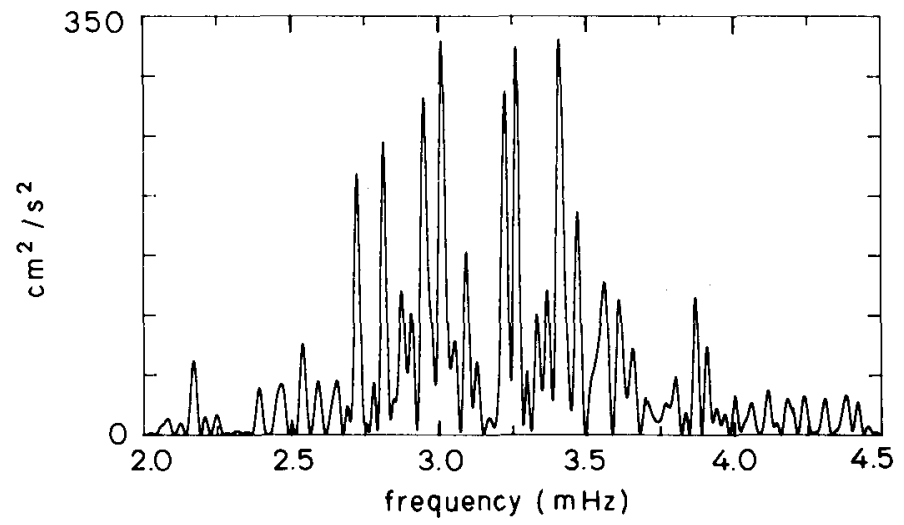

Fig. 3. Power spectrum of data illustrated in Figure 2b. The widths of the peaks are the natural widths corresponding to the 10 hours of data.

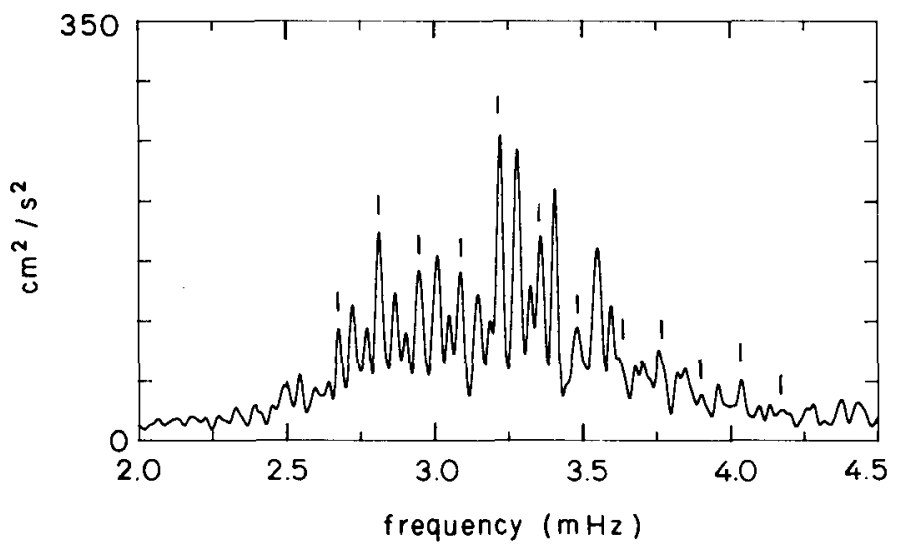

Fig. 4. Average of all power spectra taken from residual data of the type shown in Figure $2 b$ collected during the intervals listed in Table I. The vertical lines drawn above some of the peaks are the peak frequencies for oscillation modes with $l=3$ observed by Grec et al. (1983) at the South Pole. 


\section{TABLE II}

Frequencies and amplitudes of the peaks in Figure 4. The method of determining the degree $l$ of each mode is described in the text; the order $n$ was then obtained by comparison with eigenfrequencies of the solar model. The last column is the rms surface velocity amplitude per mode, computed under the assumption that the spectrum line shift measured is a pure Doppler shift associated with the line-of-sight velocity of oscillation.

\begin{tabular}{|c|c|c|c|c|}
\hline$n$ & $l$ & $\begin{array}{l}\text { Frequency } \\
(\text { microHz) }\end{array}$ & $\begin{array}{l}\text { Amplitude } \\
\left(\mathrm{cm}^{2} \mathrm{~s}^{-2}\right)\end{array}$ & $\begin{array}{l}\text { rms velocity } \\
\left(\mathrm{cm} \mathrm{s}^{-1}\right)\end{array}$ \\
\hline 16 & 3 & 2544 & 55.4 & 4.1 \\
\hline 16 & 4 & 2596 & 44.2 & 3.2 \\
\hline 16 & 5 & 2642 & 49.3 & 3.9 \\
\hline 17 & 3 & 2677 & 93.3 & 5.3 \\
\hline 17 & 4 & 2724 & 112.8 & 5.1 \\
\hline 17 & 5 & 2773 & 93.6 & 5.4 \\
\hline 18 & 3 & 2815 & 173.9 & 7.3 \\
\hline 18 & 4 & 2868 & 123.2 & 5.3 \\
\hline 18 & 5 & 2905 & 90.0 & 5.2 \\
\hline 19 & 3 & 2948 & 141.8 & 6.6 \\
\hline 19 & 4 & 3010 & 154.4 & 6.0 \\
\hline 19 & 5 & 3050 & 105.0 & 5.7 \\
\hline 20 & 3 & 3088 & 140.9 & 6.6 \\
\hline 20 & 4 & 3148 & 121.7 & 5.3 \\
\hline 20 & 5 & 3189 & 100.3 & 5.5 \\
\hline 21 & 3 & 3221 & 254.0 & 8.8 \\
\hline 21 & 4 & 3279 & 242.5 & 7.5 \\
\hline 21 & 5 & 3325 & 128.9 & 6.3 \\
\hline 22 & 3 & 3359 & 170.8 & 7.2 \\
\hline 22 & 4 & 3407 & 209.9 & 7.0 \\
\hline 23 & 3 & 3482 & 94.3 & 5.4 \\
\hline 23 & 4 & 3551 & 160.4 & 6.1 \\
\hline 23 & 5 & 3597 & 112.3 & 5.9 \\
\hline 24 & 3 & 3623 & 68.9 & 4.6 \\
\hline 24 & 4 & 3678 & 63.1 & 3.8 \\
\hline 25 & 3 & 3703 & 66.8 & 6.8 \\
\hline 25 & 4 & 3759 & 75.9 & 4.8 \\
\hline 25 & 5 & 3820 & 57.5 & 3.6 \\
\hline 26 & 2 & 3846 & 61.0 & 6.5 \\
\hline 26 & 3 & 3902 & 38.6 & 3.4 \\
\hline 26 & 4 & 3958 & 47.5 & 3.3 \\
\hline 26 & 5 & 4007 & 29.5 & 3.0 \\
\hline 27 & 3 & 4036 & 50.8 & 3.9 \\
\hline 27 & 4 & 4097 & 28.5 & 2.6 \\
\hline 27 & 5 & 4133 & 29.6 & 3.0 \\
\hline 28 & 3 & 4175 & 25.5 & 2.8 \\
\hline 28 & 4 & 4280 & 30.3 & 3.0 \\
\hline 29 & 2 & 4258 & 26.8 & 2.5 \\
\hline
\end{tabular}


Fossat et al. (1983) as $p$ modes with $l=3$. Table II lists the frequencies of the maxima of all the peaks in Figure 4, to a precision of $1 \mathrm{microHz}$.

For $l=3$ the rms difference between the peak frequencies of Fossat, Grec, and Pomerantz observed at the South Pole and those reported here observed at Stanford is $3.3 \mathrm{microHz}$. This gives an estimate of the uncertainty of our tabulated peak frequencies of the $l=3$ modes.

The overall properties of the mean spectrum can be investigated by computing its auto-correlation. This is shown on Figure 5a. It has fairly sharp peaks centered on lags of 135.5 and $271.0 \mathrm{microHz}$, corresponding to the uniform spacing of modes of like degree. These are separated by broader asymmetrical features whose maxima are at about 56 and $191 \mathrm{microHz}$. For comparison we have found auto-correlations of simulated power spectra computed as in Christensen-Dalsgaard and Gough (1982), using all the modes of model 1 of Christensen-Dalsgaard (1982) with $l$ between 0 and 20 and frequencies between 2.2 and $4.2 \mathrm{mHz}$; the observing intervals listed in Table I were used. Before computing the auto-correlation the average spectra were multiplied by $A(v)$. The results are shown in Figure $5 \mathrm{~b}$. The continuous curve was obtained using the spatial filters computed by Christensen-Dalsgaard and Gough (1982) for the Stanford observations and assuming that the modes preserve their amplitudes and phases for 5 days at a time; the well-defined maxima at lags of $46,92,138 \ldots$ micro $\mathrm{Hz}$ reflect the three-peak structure shown in Figure 1b. This is qualitatively different from the auto-correlation of the spectrum of the observations.

To try to account for this difference we have made two additional simulations. The dashed curve was obtained by assuming that the modes preserve their phases and amplitudes over the entire observing interval; this is consistent with the estimates of the coherence times quoted by Claverie et al. $(1981 \mathrm{a}, \mathrm{b})$. Values of the auto-correlation are generally lower than in the previous case, and there are fewer peaks. The dominant feature is a peak at $139 \mathrm{microHz}$. The reason for the changed appearance of the curve is that now there is no averaging over different sets of random amplitudes and phases, and so the spectrum is less regular. The second simulation used filters computed as in Christensen-Dalsgaard and Gough (1982), but assumed a central disk of radius $0.6 R_{\odot}$ and an outer annulus between 0.65 and $0.9 R_{\odot}$. The spectrum is then dominated by modes with lower $l$ : principally $l=3$ and the closely spaced pair $l=2,4$. A coherence time of 5 days was assumed. The resulting auto-correlation, shown as a dot-dashed line in Figure $5 \mathrm{~b}$, reflects the basic double-peaked structure of the spectrum. Evidently a similar result would be obtained with our Stanford filters if modes with one of the principal values of $l$ were selectively excited to a lower amplitude than the other. A glance at Figure 4 or Table II reveals that where the amplitude is high there is a tendency for every third peak (which we infer is associated with $l=5$ ) to be weak. It is evident that an increase in coherence time and a change in the sensitivity that leads to the domination of fewer modes both give better agreement with the observed autocorrelation, the latter probably being the more successful. In fact, although the dimensions of the observing apertures are known accurately, the spatial filters computed are rather uncertain, owing to the neglect of variations in the line profile over the disk of the Sun and the effects 

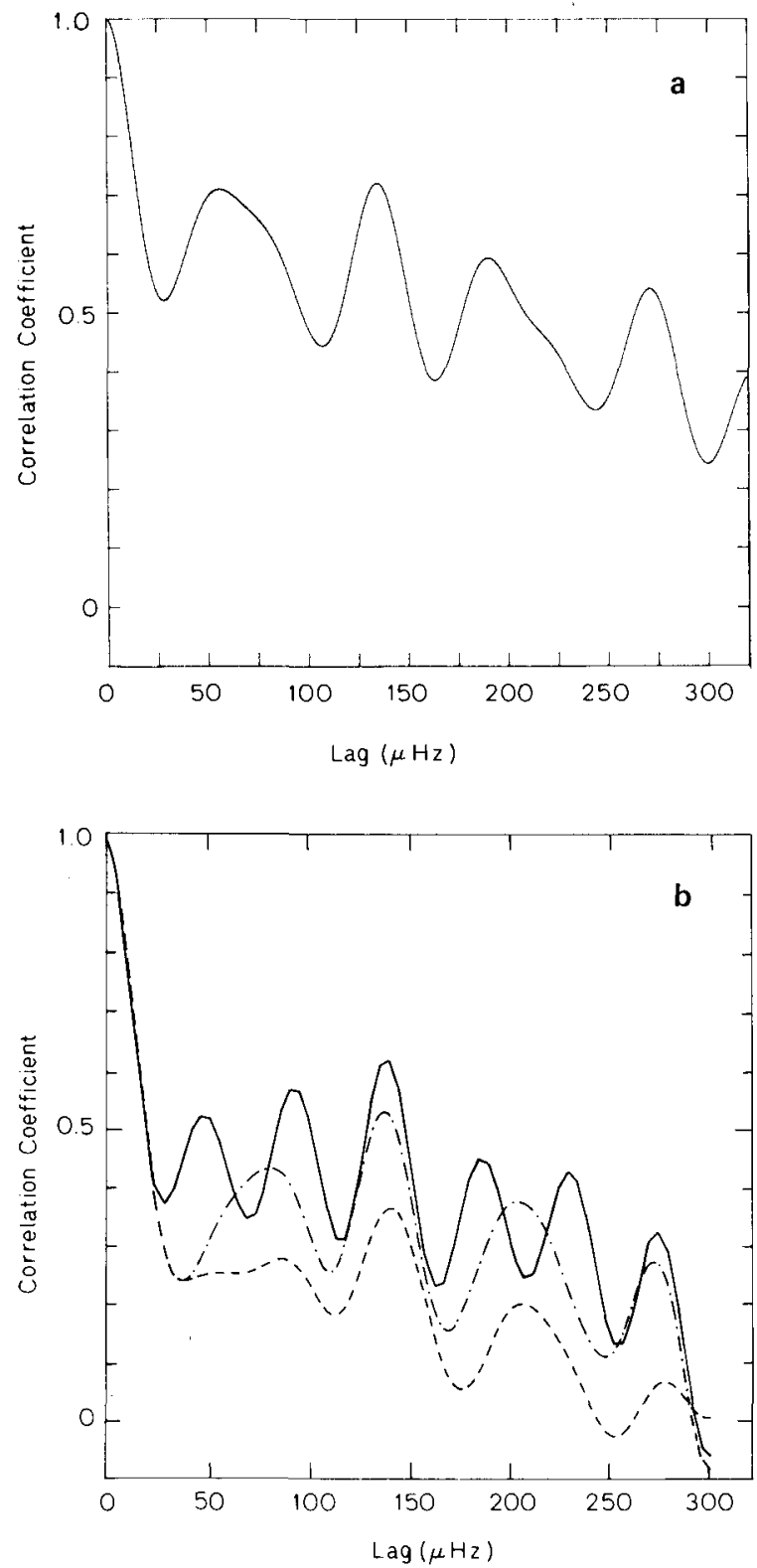

Fig. 5. Autocorrelation of power spectra: (a) of the mean spectrum shown in Figure 4, (b) of simulated mean spectra. In (b), the continuous curve represents simulated data with coherence times of 5 days, using Stanford filters; the dashed curve is similar, but with perfect phase coherence throughout the duration of the simulated observations; the dot-dashed curve represents data obtained with filters that give more weight to the modes of low degree, as described in the text.

of rotation; changes to the spatial filters would also occur if the observed line shift were not caused solely by Doppler displacements. Thus further investigations of the sensitivity of the observations are needed. Nevertheless, the autocorrelations of the spectrum of 


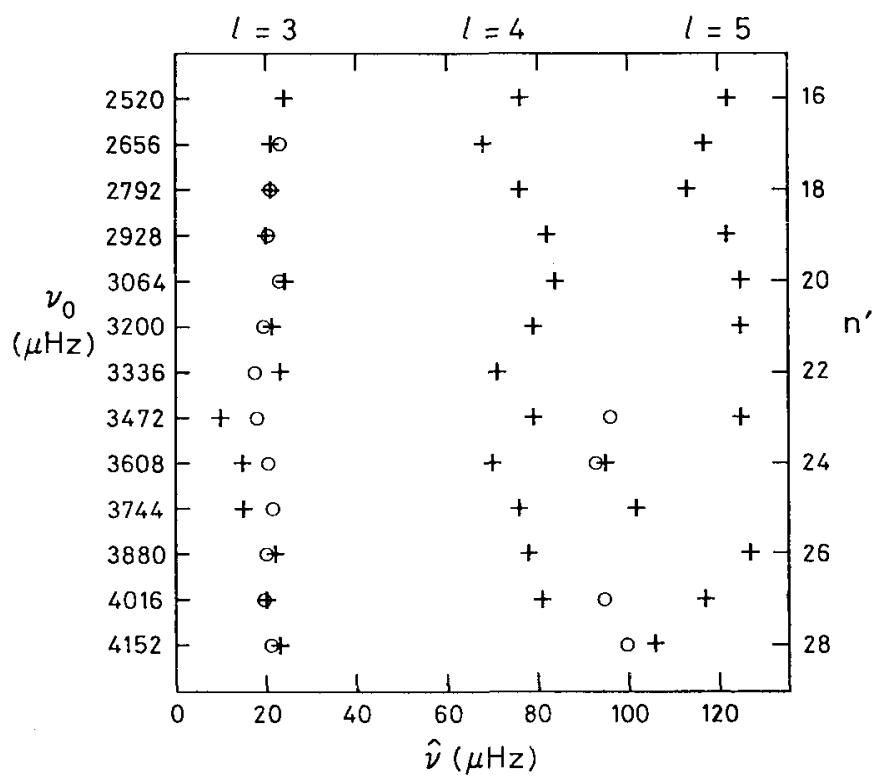

Fig. 6. Echelle diagram of the frequencies between 2.5 and $4.3 \mathrm{mHz}$ of low-degree modes, constructed with $\Delta v=136.0$ microHz. The frequencies $v$ of the modes represented in the diagram are given by $v=v_{0}+\hat{v}$. Crosses indicate the frequencies of the peaks in Figure 4. Circles represent octupole modes (with $\hat{v}$ near $20 \mathrm{microHz}$ ) and some dipole modes (with $\hat{v}$ near $100 \mathrm{microHz}$ ) observed from the South Pole by Grec et al. (1983). Thus by comparing our results with the South-Pole data and with theory we identify the degrees of the modes responsible for the three principal columns; these are indicated at the top of the diagram. We presume the frequencies 3703,3846 , and $4258 \mathrm{microHz}$ to correspond to dipole modes. The ordinate scale $n^{\prime}$ on the right is the order $n$ of modes with $l \geq 3$, and is $n-1$ when $l=2$. This identification was obtained by the least-squares comparison with theory described in the text. Fitting regression lines $\alpha+\beta\left(n-n_{0}\right)$, with $n_{0}=22-\frac{1}{2} l$, to the three principal columns of Stanford frequencies $v$ yields $(\alpha, \beta)=(3152 \pm 1$, $135.7 \pm 0.3),(3140 \pm 1,136.2 \pm 0.4)$, and $(3117 \pm 1,136.5 \pm 0.3)$ for $l=3,4$, and 5 , respectively.

the observations, in particular the peak at $135.5 \mathrm{microHz}$, demonstrates clearly the presence of resolved five-minute oscillations.

Figure 6 is a plot of the reduced frequencies $\hat{v}$ associated with the frequencies listed in Table II, computed on the frequency interval $\Delta v=136.0 \mathrm{microHz}$. Following Grec (1981), $\hat{v}$ has been plotted at different heights on the diagram in decreasing order of the multiple of $\Delta v$ that had been subtracted from $v$, as in an echelle spectrum. On the whole, the frequencies form three distinct almost vertical columns, as we anticipated. Included in the figure are the frequencies of the octupole modes identified by Grec et al. (1983) in the Antarctic data, and also some of the dipole modes. The former almost coincide with our first column of modes, which we therefore identify with $l=3$. On consulting Figure $1 \mathrm{~b}$, we infer that the remaining two columns are produced by modes with $l=4$ and $l=5$. We also have evidence for quadrupole modes lying between the $l=4$ and $l=5$ columns.

Our columns are not as smooth as those of Grec et al. The reason is probably that our results are more prone to interference from other modes. The presence of a mode of lower amplitude whose frequency is too close to one of the principal modes for the 
separation to be resolved has the effect of shifting the line in the power spectrum associated with the principal mode. We conjecture that the break in the octupole column at $3482 \mathrm{microHz}$, for example, is due to the intersection of the locus of the octupole modes with the locus of modes with $l=9$.

\section{Comparison with Previous Observations}

In almost all cases our octupole frequencies are in good agreement with those reported by Grec et al. (1983). We can make little direct comparison with the other observations of five-minute modes because, aside from our few quadrupole modes, we have not observed modes of the same degree. However, an indirect comparison is possible.

It is a property of the modes under consideration that $n \gg l$. When this condition is satisfied, the frequencies $v$ are roughly proportional to $n+\frac{1}{2} l$. The asymptotic analysis by Tassoul (1980) shows that to a higher approximation

$$
v \sim\left(n+\frac{1}{2} l+\varepsilon\right) v_{0}-[l(l+1)+\delta] A v_{0}^{2} / v,
$$

where

$$
v_{0}=\left(2 \int_{0}^{R} C^{-1} \mathrm{~d} r\right)^{-1},
$$

$R$ is the radius of the Sun, $c(r)$ is the sound speed and the quantities $\varepsilon, A$ and $\delta$ are other constants of the equilibrium model. Thus the deviation from the simple linear approximation to $v$ is a linear function of $y \equiv l(l+1)$. Introducing $x=n+\frac{1}{2} l$ one can approximate (6.1) in the neighbourhood of $x=x_{0}$, where $x_{0}$ is constant, by

$$
v \simeq \alpha+\beta\left(x-x_{0}\right) .
$$

Then

$$
\alpha \simeq v_{0}\left(X+A \delta X^{-1}+A X^{-1} y\right)
$$

and

$$
\beta \simeq v_{0}\left(1-A \delta X^{-2}-A X^{-2} y\right),
$$

where $X=x_{0}+\varepsilon$. Thus, to the order of accuracy of (6.1), $\alpha$ and $\beta$ should vary linearly with $y$, and indeed we have found that to be approximately true of numerically computed eigenfrequencies of realistic solar models. For Model 1 of Christensen-Dalsgaard (1982), for example, $\mathrm{d} \alpha / \mathrm{d} y \simeq-1.76 \mu \mathrm{Hz}$ and $\mathrm{d} \beta / \mathrm{d} y \simeq 0.050 \mu \mathrm{Hz}$ when $x_{0}=22$.

We have computed estimates of $\alpha$ and $\beta$ for the Sun for each value of $l$ separately by linear regression with our own data and all the other published observations that have succeeded in distinguishing modes of different degree. The results are shown in Figure 7. To aid comparison, lines with slopes $-1.76 \mu \mathrm{Hz}$ and $0.050 \mu \mathrm{Hz}$ are included. The absolute positions of these lines have been chosen to pass between the data; they do not agree precisely with the numerical results, partly because the solar model used to 

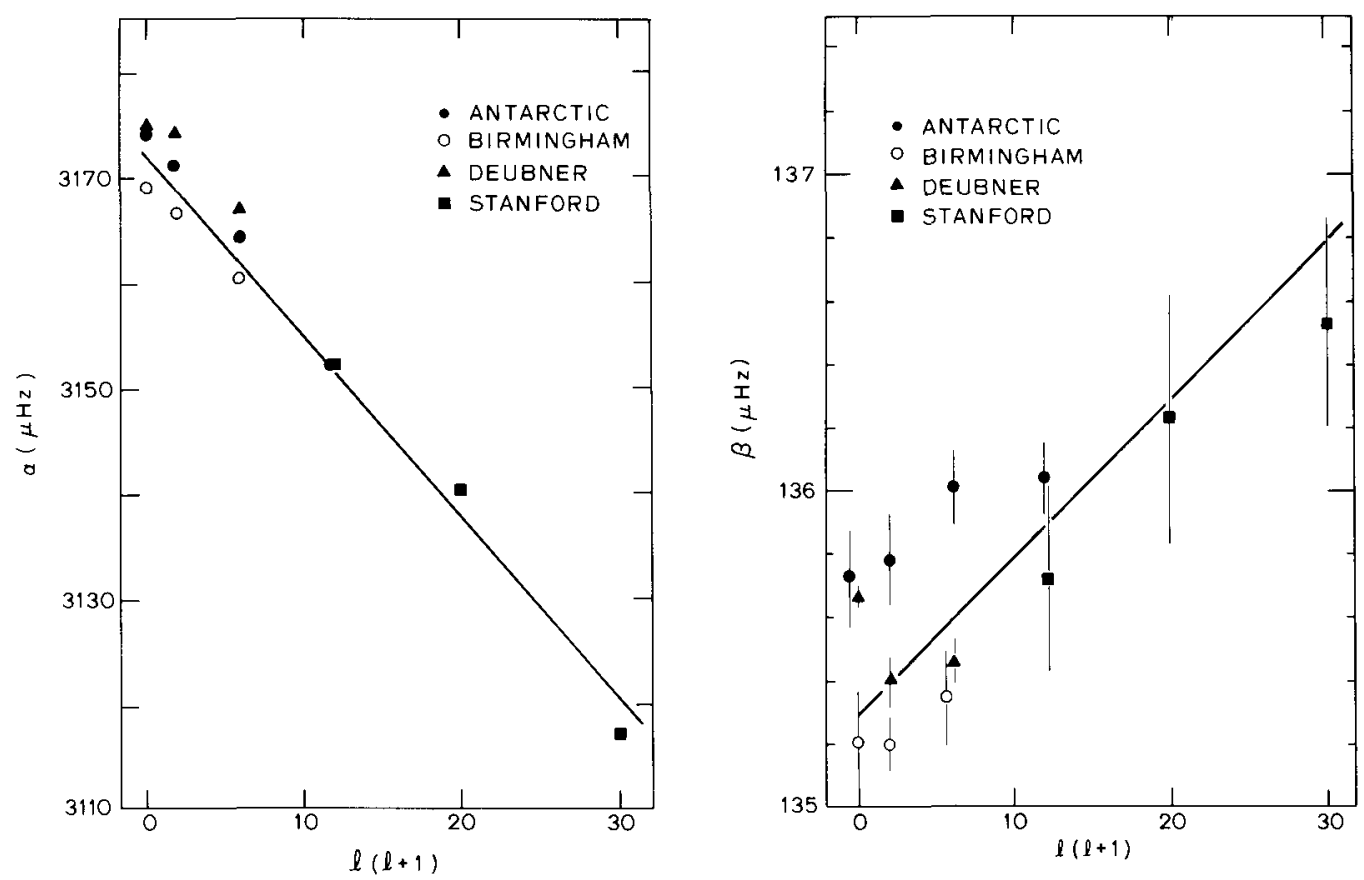

Fig. 7. Regression coefficients: (a) $\alpha$, (b) $\beta$ computed with $x_{0}=22$. Squares represent the Stanford results reported in this paper, filled circles the Antarctic data of Grec et al. (1983), open circles the Birmingham data of Claverie et al. (1981 a, b) and triangles the data of Deubner (1981). The recent SMM-ACRIM data of Woodard and Hudson (1983) agree very well with those of Claverie et al. (1981a, b). The standard errors in $\alpha$ are roughly the size of the symbols; those of $\beta$ are indicated by vertical lines. Where two data overlap, they have been shifted slightly to the left or right. The diagonal lines in (a) and (b) have slopes $-1.75 \mathrm{microHz}$ and $0.050 \mathrm{microHz}$, respectively.

compute the eigenfrequencies appears to be somewhat helium deficient (cf. ChristensenDalsgaard and Gough, 1980, 1981). It is evident that our results are roughly in accord with the others, though the differences between the observations are greater than the standard errors. Part, though not all, of these differences results from having analysed data over different ranges of frequency.

\section{Conclusion}

We have observed and identified solar oscillations with $l=3,4,5$ in the frequency range $2.5-4.3 \mathrm{mHz}$. The frequencies of the octupole modes are in good agreement with those found by Grec et al. (1983), and with the help of a theoretical extrapolation we have demonstrated fair agreement with other observations that have been published. There are, however, systematic discrepancies between the observations of modes with $l \leq 2$. These discrepancies are not insubstantial, if interpretated in terms of the differences in the internal structure of the sun that they seem to imply. 


\section{Acknowledgements}

We thank E. Fossat, G. Grec, and M. Pomerantz for communicating to us their observations prior to publication, and E. Fossat for suggesting the format of Figure 6 . This work was supported in part by the Office of Naval Research under Contract N00014-76-C-0207, by the National Aeronautics and Space Administration under Grant NGR 05-020-559 and Contract NAS5-24420, by the Division of Atmospheric Sciences, Solar Terrestrial Research Program of the National Science Foundation under Grant ATM77-20580, by the Max C. Fleischmann Foundation, by the National Center for Atmospheric Research, which is sponsored by the National Science Foundation, and by the Science and Engineering Research Council.

\section{References}

Babcock, H. W.: 1953, Astrophys. J. 118, 387.

Christensen-Dalsgaard, J.: 1980, in P. Ledoux (ed.), Proc. 5th European Meeting in Astronomy, Institute d'Astrophysique, Liège, p. A.1.1.

Christensen-Dalsgaard, J.: 1982, Monthly Notices Roy. Astron. Soc. 199, 735.

Christensen-Dalsgaard, J. and Gough, D. O.: 1980, Nature 288, 544.

Christensen-Dalsgaard, J. and Gough, D. O.: 1981, Astron. Astrophys. 104, 173.

Christensen-Dalsgaard, J. and Gough, D. O.: 1982, Monthly Notices Roy. Astron. Soc. $198,141$.

Claverie, A., Isaak, G. R., McLeod, C. P., van der Raay, H. B., and Roca Cortes, T.: 1979, Nature $282,591$.

Claverie, A., Isaak, G. R., McLeod, C. P., van der Raay, H. B., and Roca Cortes, T.: 1980, Astron. Astrophys. 91, L9.

Claverie, A., Isaak, G. R., McLeod, C. P., van der Raay, H. B., and Roca Cortes, T.: 1981a, Nature 293, 443. Claverie, A., Isaak, G. R., McLeod, C. P., van der Raay, H. B., and Roca Cortes, T.: 1981b, Solar Phys. 74, 51 .

Deubner, F.-L.: 1981, Nature 290, 682.

Dittmer, P. H.: 1978, Astrophys. J. 224, 265.

Fossat, E., Grec, G., and Pomerantz, M.: 1981, Solar Phys. 74, 59.

Grec, G.: 1981, These de Dr Sci. Phys., Université de Nice.

Grec, G., Fossat, E., and Pomerantz, M.: 1980, Nature 288, 541.

Grec, G., Fossat, E., and Pomerantz, M.: 1983, Solar Phys. 82, 55 (this volume).

Severny, A. B., Kotov, V. A., and Tsap, T. T.: 1976, Nature 259, 87.

Severny, A. B., Kotov, V. A., and Tsap, T. T.: 1981, Solar Phys. 74, 65.

Tassoul, M.: 1980, Astrophys. J. Suppl. 43, 469.

Woodard, M. and Hudson, H.: 1983, Solar Phys. 82, 67 (this volume). 